\title{
Plasma Bmi1 mRNA as a potential prognostic biomarker for distant metastasis in colorectal cancer patients
}

\author{
JASON CHUN-SANG PUN ${ }^{1 *}$, JOYCE YEE-JING CHAN ${ }^{1 *}$, BOBBY KA-MING CHUN $^{1}$, KA-WAI NG ${ }^{1}$, \\ SAMUEL YUNG-KIN TSUI ${ }^{1}$, TIMOTHY MING-HUN WAN ${ }^{2}$, OSWENS LO ${ }^{2}$, \\ JENSEN TUNG-CHUNG POON ${ }^{2}$, LUI NG $^{2}$ and ROBERTA PANG ${ }^{2,3}$ \\ ${ }^{1}$ The Chinese Foundation Secondary School, Hong Kong; ${ }^{2}$ Department of Surgery and ${ }^{3}$ Centre for Cancer Research, \\ Li Ka Shing Faculty of Medicine, The University of Hong Kong, Hong Kong, SAR, P.R. China
}

Received January 31, 2014; Accepted April 1, 2014

DOI: $10.3892 / \operatorname{mco} .2014 .321$

\begin{abstract}
Bmil is overexpressed in gastrointestinal cancers, including colorectal cancer (CRC); however, its role as a non-invasive biomarker in CRC has not been established. The aim of this study was to compare the plasma Bmil mRNA levels prior to and following curative resection of the primary tumor in CRC patients and to determine their association with the clinicopathological parameters. The plasma Bmi1 mRNA level was measured by quantitative polymerase chain reaction and expressed as cycle threshold value. There was no significant difference between the overall pre- and postoperative plasma Bmil mRNA level $(31.73 \pm 2.63$ vs. $31.93 \pm 2.88$, respectively; $\mathrm{P}=0.614)$ in $45 \mathrm{CRC}$ patients. However, when grouped into non-metastatic and metastatic CRC patients, the postoperative Bmil transcript level was found to be significantly lower compared to the preoperative level in patients with non-metastatic CRC (32.13 $\pm 2.67731 .44 \pm 2.764$, respectively; $\mathrm{P}=0.041)$, whereas there was a trend towards a higher postoperative Bmil transcript level compared to the preoperative level in the metastatic counterpart $(30.85 \pm 3.916$ vs. $33.27 \pm 0.718$, respectively; $\mathrm{P}=0.164)$. Furthermore, when the patients were categorized into two groups according to their plasma Bmil postoperative vs. preoperative level status, we observed that patients without a reduction in the postoperative plasma Bmi1 mRNA levels exhibited a significantly higher rate of distant metastasis following primary resection $(\mathrm{P}=0.017)$ and a significantly worse prognosis regarding disease-free survival $(\mathrm{P}=0.016)$ when compared to the reduced postoperative plasma Bmil level counterparts. In conclusion, plasma Bmil mRNA
\end{abstract}

Correspondence to: Dr Roberta Pang, Department of Surgery and Center for Cancer Research, Li Ka Shing Faculty of Medicine, The University of Hong Kong, 21 Sassoon Road, Pokfulam, Hong Kong, SAR, P.R. China

E-mail: robertap@hku.hk

${ }^{*}$ Contributed equally

Key words: Bmil, colorectal cancer, metastasis levels may serve as a non-invasive biomarker for monitoring occult metastasis and predicting the development of distant metastasis.

\section{Introduction}

Colorectal cancer (CRC) is the third most commonly diagnosed cancer in males and the second in females, with $>1.2$ million new cancer cases and 608,700 deaths estimated to have occurred in 2008 (1). Recent advances in therapeutic strategies and surgical techniques have significantly improved the prognosis of CRC patients with primary disease; however, metastasis is a major concern for CRC patients and physicians. Thirty-five percent of CRC patients have metastatic tumors at the time of diagnosis and 33-50\% of the patients without metastases will progress to stage IV during the course of their disease $(1,2)$. Hence, it is necessary to develop prominent biomarkers for detecting the presence of occult metastasis, as well as for predicting the development of future metastasis, which may provide a useful reference for the therapeutic management of CRC patients.

Potential stem cell marker, Bmil, is a member of the polycomb-repressive complex 1 , with a key role in gene silencing through chromatin modifications $(3,4)$. In addition to the proposed role for Bmil as a key regulator of cell growth control/senescence mechanisms, accumulating evidence supports the role of Bmil in tumorigenesis. Bmil is overexpressed in a variety of human cancers and its overexpression was found to be correlated with tumor progression in CRC (5-7) and other gastrointestinal cancers, including esophageal squamous cell carcinoma $(8,9)$, pancreatic cancer $(10)$ and gastric cancer (11), indicating its functional and prognostic role in gastrointestinal cancer patients. The Bmil autoantibody in the serum has also been suggested as a minimally invasive biomarker for the prognosis of nasopharyngeal (12), esophageal squamous cell (8) and cervical carcinoma (13). More importantly, circulating Bmil mRNA has been detected in the plasma and was correlated with poor prognosis of advanced breast (14) and uterine cervical cancer patients (15). Those studies demonstrated the potential of Bmil as a non-invasive surrogate marker for a variety of cancer patients; however, such an application in CRC has not yet been investigated. 
In this study, the clinical significance of plasma Bmil mRNA in CRC patients was investigated. The pre- and postoperative plasma Bmil mRNA levels in CRC patients were determined by quantitative polymerase chain reaction (PCR) and correlated with the clinicopathological parameters, in order to determine whether monitoring of plasma Bmil in CRC patients is predictive of the development of distant metastasis.

\section{Materials and methods}

Patients and plasma samples. Blood samples were collected from 45 patients who underwent surgical resection of primary CRC during their follow-up at the Department of Surgery, Queen Mary Hospital, The University of Hong Kong, between 2009 and 2013. Among these 45 patients, who presented with no evidence of distant metastasis at the time of the primary resection, 6 developed distant metastasis within 3 years, whereas the remaining patients were recurrence-free at the last follow-up visit to our clinic. The eligibility criteria for this study included pre- and postoperative plasma sample availability for quantitative PCR. Blood was anticoagulated by EDTA and centrifuged at $1,500 \mathrm{~g}$ for $10 \mathrm{~min}$. The plasma was collected, divided into aliquots and snap-frozen at $-80^{\circ} \mathrm{C}$ until use.

This study was approved by the Institutional Review Board of our hospital and the patients provided written informed consent prior to inclusion.

RNA isolation, reverse transcription and quantitative PCR. RNA was isolated from $250 \mu \mathrm{l}$ of plasma sample using the mirVana ${ }^{\mathrm{TM}}$ miRNA isolation kit (Life Technologies, Carlsbad, CA, USA), according to the manufacturer's instructions. For the synthesis of first-strand cDNA, $8 \mu 1$ RNA was reversed transcribed using PrimeScript ${ }^{\mathrm{TM}}$ RT Master mix (Takara Bio, Inc., Shiga, Japan) in accordance with the manufacturer's instructions. Quantitative PCR was performed in a final volume of $15 \mu \mathrm{l}$ containing $1.5 \mu \mathrm{l}$ RT transcript, $0.2 \mu \mathrm{M}$ of each primer, 1X ROX reference dye and $7.5 \mu \mathrm{l}$ of FastStart Universal SYBR-Green Master (ROX) (Roche Applied Science, Indianapolis, IN, USA). The Bmil forward and reverse primer sequences (5'-3') were ATCCCCACCTGATGTGTG and AAAGCCCTGGAACTAATTTG, respectively. Quantitative PCR was performed using the ABI 7900HT Fast Real-Time PCR system (Applied Biosystems, Foster City, CA, USA) at $95^{\circ} \mathrm{C}$ for $10 \mathrm{~min}$, followed by 40 cycles at $95^{\circ} \mathrm{C}$ for $15 \mathrm{sec}$ and at $56^{\circ} \mathrm{C}$ for $1 \mathrm{~min}$.

Statistical analysis. The plasma Bmil mRNA level was expressed as the cycle threshold $(\mathrm{Ct})$ detected by quantitative PCR using the same threshold value of fluorescent signal intensity. The difference in plasma Bmil mRNA level between the two different groups was evaluated by the Student's t-test, whereas the difference between the pre- and postoperative blood levels in the same patient was evaluated by the paired t-test. The differences in recurrence rates among different groups of CRC patients were evaluated by the Fisher's exact test. The disease-free survival was analyzed using the Kaplan-Meier product limit method and the log-rank test. All the statistical analyses were performed with SigmaPlot software, version 10.0 (Systat Software Inc., San Jose, CA, USA).

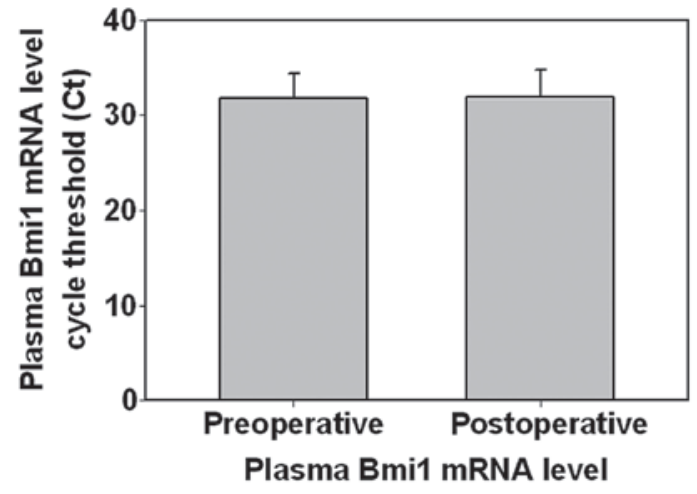

Figure 1. Plasma Bmil transcript level prior to and following surgical resection of primary colorectal cancer in 45 patients. There was no significant difference $(\mathrm{P}=0.614)$ between the pre- $(31.73 \pm 2.63)$ and postoperative $(31.93 \pm 2.88)$ Bmil transcript levels (expressed as mean cycle threshold (Ct) \pm standard deviation).

$\mathrm{P}<0.05$ was considered to indicate a statistically significant difference.

\section{Results}

Correlation of pre- and postoperative plasma Bmil $\mathrm{mRNA}$ levels with clinicopathological parameters. We first compared the plasma Bmil transcript level prior to and following surgical resection of primary colorectal tumor in 45 patients. As shown in Fig. 1, the pre- and postoperative Bmil transcript levels (expressed as mean $\mathrm{Ct} \pm$ standard deviation, i.e., the higher the $\mathrm{Ct}$, the lower the transcript level) were $31.73 \pm 2.63$ and $31.93 \pm 2.88$, respectively, without a statistically significant difference between the two levels $(\mathrm{P}=0.614)$. We further compared the pre- and postoperative Bmil transcript levels in patients with different clinicopathological parameters (Table I). There were no significant changes in pre- and postoperative plasma Bmil transcript levels in CRC patients of different age, gender, lymph node status and TNM stage. However, the changes were significantly different between patients with metastatic and non-metastatic CRC. In the 39 non-metastatic CRC patients, the postoperative Bmil transcript level was significantly lower compared to the preoperative level $(32.13 \pm 2.677$ vs. $31.44 \pm 2.764$, respectively; $\mathrm{P}=0.041)$. Furthermore, in the $6 \mathrm{CRC}$ patients who developed metastasis, there was a trend for higher postoperative Bmil transcript level compared to the preoperative level ( $30.85 \pm 3.916$ vs. $33.27 \pm 0.718$, respectively; $\mathrm{P}=0.164)$. These results suggested that an increase in the postoperative Bmil level correlated with the development of metastasis in CRC patients.

Due to sampling limitations, the postoperative blood plasma specimens were obtained from CRC patients at different time points following surgery (range, 0.5-25 months; median, 5 months). To determine whether the length of time post-operation affects the plasma Bmil levels, we analyzed the association between the length of time post-operation and the difference between post- and preoperative plasma Bmil levels in CRC patients. Our results demonstrated that there was no significant correlation ( $r=-0.0116, P=0.945)$, suggesting that the length of time post-operation does not affect the results obtained. 
Table I. Clinicopathological correlation of plasma Bmil mRNA levels in colorectal cancer patients.

\begin{tabular}{lccc}
\hline Clinico- & \multicolumn{3}{c}{ Plasma Bmi1 mRNA level } \\
pathological & No. of & pre- vs. postoperarive \\
characteristics & cases $^{\text {a }}$ & (mean Ct \pm SD) & P-value
\end{tabular}

\begin{tabular}{llll}
\hline $\begin{array}{l}\text { Age (years) } \\
<65\end{array}$ & 12 & $29.82 \pm 1.76$ vs. $30.07 \pm 2.48$ & 0.753 \\
$\geq 65$ & 24 & $30.36 \pm 2.22$ vs. $30.18 \pm 2.97$ & 0.718 \\
Gender & & & \\
Male & 22 & $30.33 \pm 2.01$ vs. $30.04 \pm 3.04$ & 0.657 \\
Female & 14 & $29.96 \pm 2.21$ vs. $30.29 \pm 2.42$ & 0.471
\end{tabular}

Tumor size

(cm)

$\begin{array}{lccc}<5 & 15 & 30.48 \pm 2.32 \text { vs. } 29.99 \pm 3.20 & 0.523 \\ \geq 5 & 9 & 30.18 \pm 2.16 \text { vs. } 30.49 \pm 2.70 & 0.589\end{array}$

Lymph node metastasis

$\begin{array}{llll}\text { Absent } & 17 & 30.04 \pm 2.14 \text { vs. } 30.57 \pm 2.34 & 0.224 \\ \text { Present } & 15 & 30.45 \pm 2.27 \text { vs. } 29.77 \pm 3.49 & 0.439 \\ \text { TNM stage } & & & \\ \text { I-II } & 15 & 30.68 \pm 1.76 \text { vs. } 30.90 \pm 2.14 & 0.524 \\ \text { III-IV } & 12 & 30.14 \pm 2.39 \text { vs. } 29.31 \pm 3.76 & 0.457\end{array}$

Distant metastasis

$\begin{array}{lccc}\text { Absent } & 32 & 31.44 \pm 2.76 \text { vs. } 32.13 \pm 2.68 & 0.041 \\ \text { Present } & 6 & 33.27 \pm 0.72 \text { vs. } 30.85 \pm 3.92 & 0.164\end{array}$

${ }^{a}$ The total number of cases is $<45$ due to incomplete information. $\mathrm{Ct}$, cycle threshold; SD, standard deviation.

Association of higher postoperative plasma Bmil mRNA with the development of distant metastasis. We next categorized the 45 patients into two groups according to their plasma Bmi 1 post- vs. preoperative level status (Table II). Of the 45 patients, 29 exhibited a reduced postoperative Bmil level compared to the preoperative level, whereas 16 displayed increased or unchanged Bmil level following resection of the primary tumor. Of the 16 patients who exhibited no reduction in Bmil level, 5 developed metastasis within 36 months post-operation. By contrast, only 1 of the 29 patients exhibiting lower postoperative Bmil level developed metastasis, suggesting that patients with reduced postoperative Bmil levels were less likely to develop metastasis $(\mathrm{P}=0.017)$.

Finally, the prognostic significance of the changes in the post- vs. preoperative Bmil level in CRC patients was asessed (Fig. 2). The patients with a reduced postoperative Bmil level $(n=29)$ had a significantly better prognosis $(\mathrm{P}=0.016)$ for disease-free survival when compared to their increased postoperative Bmil level counterparts $(n=16)$, further demonstrating the correlation of changes in pre- and postoperative Bmil levels with the development of metastasis.

Our results suggested that reduced plasma Bmil transcript levels following resection of the primary tumor was a good prognostic factor for CRC patients, whereas patients with an
Table II. Plasma Bmil mRNA level changes following curative resection of the primary tumor predict development of metastasis.

\begin{tabular}{lcc}
\hline & \multicolumn{2}{c}{ Plasma Bmil mRNA level } \\
\cline { 2 - 3 } & Post- $>$ preoperative & Post- $<$ preoperative \\
\hline Metastatic & 5 & 1 \\
Non-metastatic & 11 & 28 \\
& Fisher's exact test: $\mathrm{P}=0.017$ \\
\hline
\end{tabular}

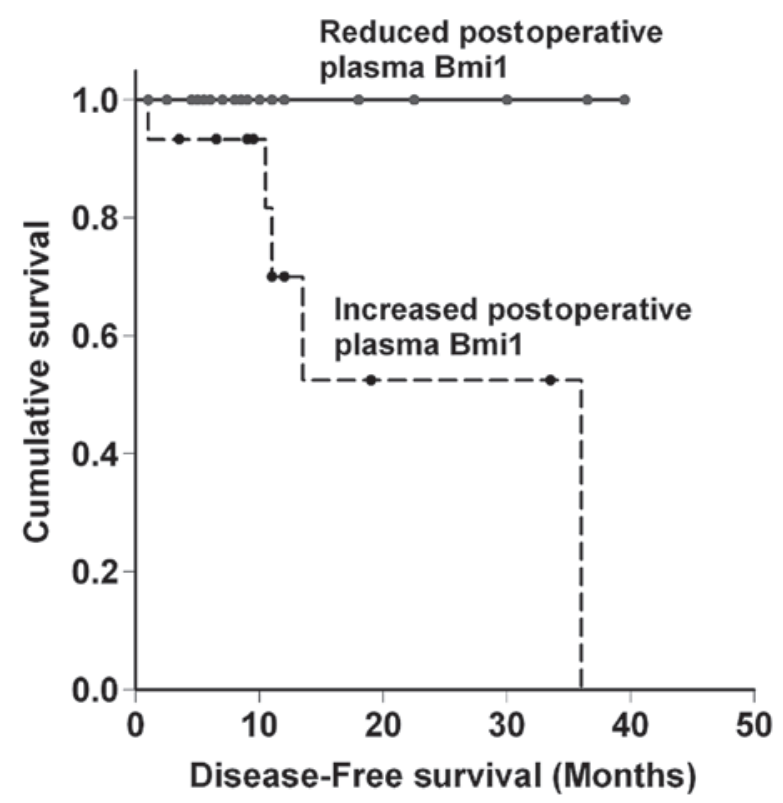

Figure 2. Prognostic significance of changes in post- vs. preoperative Bmil levels in colorectal cancer patients. Patients with a decreased postoperative plasma Bmil level $(n=29)$ had a significantly better prognosis $(P=0.016)$ for disease-free survival when compared to those with increased plasma postoperative Bmil levels $(\mathrm{n}=16)$.

increased Bmil level were more likely to develop postoperative metastasis. Therefore, monitoring the changes in the plasma Bmil level is a potential prognostic biomarker for the optimal management of CRC patients following curative resection of the primary tumor.

\section{Discussion}

Circulating Bmil mRNA prior to any treatment has been suggested as a surrogate marker of poor prognosis in patients with breast (14) and uterine cervical cancer (15). In our model, we observed that preoperative circulating Bmil mRNA did not correlate with any clinical parameters of CRC patients (data not shown), which may be explained by the relatively small number of patients recruited in this study. However, we demonstrated the significance of monitoring the changes in plasma Bmil transcript levels prior to and following surgical resection of the primary tumor in CRC patients, suggesting that monitoring the changes in circulating Bmil mRNA levels may be a more accurate prognostic biomarker in CRC patients. 
Plasma Bmil mRNA levels in cancer patients were found to be significantly higher compared to those in normal subjects $(14,15)$, suggesting that the increased circulating Bmi1 mRNA may originate from the primary tumor and surgical resection of the primary tumor should bring down the levels of such transcripts. Of note, our study did not demonstrate such an effect. We found no significant difference between the overall pre- and postoperative plasma Bmil levels in CRC patients. Therefore, we hypothesized that a subset of CRC patients whose Bmil levels remained high following primary tumor resection accounted for such findings and we considered that those patients may be at higher risk of developing metastasis, since high Bmil levels have been associated with cancer metastasis (15-18). To test our hypothesis, we divided the CRC patients into non-metastatic and metastatic and found that the postoperative circulating Bmil mRNA level in non-metastatic patients decreased to a mean value of 0.6 -fold of the preoperative level, confirming that resection of the primary tumor removes the source of circulating Bmil mRNA. However, metastatic patients exhibited a mean of 5-fold induction in their circulating Bmil level postoperatively, which is most likely the result of dissemination of CRC tumor cells that accounted for the development of metastasis.

In the second part of our analysis, we found that monitoring the changes of plasma Bmil mRNA prior to and following curative resection was prognostic for the development of future metastasis, suggesting that circulating plasma Bmil mRNA may be used as a non-invasive biomarker for predicting and monitoring occult metastasis in CRC patients. We consider this finding to be useful for physicians in the postoperative treatment of CRC patients, such as applying a more aggressive dosage of adjuvant therapy to patients exhibiting no reduction of circulating Bmil mRNA after having their primary tumors removed.

Bmil mRNA and protein overexpression were previously demonstrated in CRC tumor $(5,7)$ and were positively correlated with tumor stage, invasion and metastasis (6), as well as with poor disease-free and overall survival $(6,19)$, suggesting that Bmil is a promising prognostic biomarker in CRC patients. To the best of our knowledge, this study was the first to further demonstrate that the changes in plasma Bmi1 mRNA level prior to and following resection of the primary tumor was prognostic for the development of metastasis, indicating the potential of circulating Bmil mRNA levels as a non-invasive, convenient and relatively cost-effective surrogate biomarker for the management of postoperative CRC patients and possibly patients with other types of cancer in which Bmil is also overexpressed.

\section{References}

1. Jemal A, Bray F, Center MM, Ferlay J, Ward E and Forman D: Global cancer statistics. CA Cancer J Clin 61: 69-90, 2011.

2. Garden OJ, Rees M, Poston GJ, et al: Guidelines for resection of colorectal cancer liver metastases. Gut 55 Suppl 3: iii1-iii8, 2006.

3. Valk-Lingbeek ME, Bruggeman SW and van Lohuizen M: Stem cells and cancer; the polycomb connection. Cell 118: 409-418, 2004.

4. Widschwendter M, Fiegl H, Egle D, et al: Epigenetic stem cell signature in cancer. Nat Genet 39: 157-158, 2007.

5. Kim JH, Yoon SY, Kim CN, et al: The Bmi-1 oncoprotein is overexpressed in human colorectal cancer and correlates with the reduced p16INK4a/p14ARF proteins. Cancer Lett 203: 217-224, 2004.

6. Li DW, Tang HM, Fan JW, et al: Expression level of Bmi-1 oncoprotein is associated with progression and prognosis in colon cancer. J Cancer Res Clin Oncol 136: 997-1006, 2010.

7. Tateishi K, Ohta M, Kanai F, et al: Dysregulated expression of stem cell factor Bmil in precancerous lesions of the gastrointestinal tract. Clin Cancer Res 12: 6960-6966, 2006.

8. Liu WL, Guo XZ, Zhang LJ, et al: Prognostic relevance of Bmi-1 expression and autoantibodies in esophageal squamous cell carcinoma. BMC Cancer 10: 467, 2010.

9. Lv J, Cao XF, Ji L, et al: Association of $\beta$-catenin, Wnt1, Smad4, Hoxa9, and Bmi-1 with the prognosis of esophageal squamous cell carcinoma. Med Oncol 29: 151-160, 2012.

10. Song W, Tao K, Li H, et al: Bmi-1 is related to proliferation, survival and poor prognosis in pancreatic cancer. Cancer Sci 101: 1754-1760, 2010.

11. Zhang XW, Sheng YP, Li Q, et al: BMI1 and Mel-18 oppositely regulate carcinogenesis and progression of gastric cancer. Mol Cancer 9: 40, 2010.

12. Tong YQ, Liu B, Huang J, et al: BMI-1 autoantibody in serum as a new potential biomarker of nasopharyngeal carcinoma. Cancer Biol Ther 7: 340-344, 2008.

13. Tong YQ, Liu B, Zheng HY, et al: BMI-1 autoantibody as a new potential biomarker for cervical carcinoma. PLoS One 6: e27804, 2011.

14. Silva J, Garcia V, Garcia JM, et al: Circulating Bmi-1 mRNA as a possible prognostic factor for advanced breast cancer patients. Breast Cancer Res 9: R55, 2007.

15. Zhang X, Wang C, Wang L, et al: Detection of circulating Bmi-1 mRNA in plasma and its potential diagnostic and prognostic value for uterine cervical cancer. Int J Cancer 131: 165-172, 2012.

16. Meng X, Wang Y, Zheng X, et al: shRNA-mediated knockdown of Bmi-1 inhibit lung adenocarcinoma cell migration and metastasis. Lung Cancer 77: 24-30, 2012.

17. Huang J, Qiu Y, Chen G, Huang L and He J: The relationship between Bmi-1 and the epithelial-mesenchymal transition in lung squamous cell carcinoma. Med Oncol 29: 1606-1613, 2012.

18. Guo BH, Feng Y, Zhang R, et al: Bmi-1 promotes invasion and metastasis, and its elevated expression is correlated with an advanced stage of breast cancer. Mol Cancer 10: 10, 2011.

19. Du J, Li Y, Li J and Zheng J: Polycomb group protein Bmil expression in colon cancers predicts the survival. Med Oncol 27: 1273-1276, 2010. 